\section{Original Article \\ Pediatrics}

Check for updates

\section{OPEN ACCESS}

Received: Aug 4, 2020

Accepted: Oct 15, 2020

Address for Correspondence: Hoon-Chul Kang, MD, PhD Department of Pediatrics, Yonsei University College of Medicine, 50-1 Yonsei-ro, Seodaemun-gu, Seoul 03722, Korea. E-mail: hipo0207@yuhs.ac

\section{Heung Dong Kim, MD, PhD}

Department of Pediatrics, Severance Children's Hospital, Yonsei University College of Medicine, 50-1 Yonsei-ro, Seodaemun-gu, Seoul 03722, Korea.

E-mail:HDKIMMD@yuhs.ac

${ }^{*}$ Chung Mo Koo and Se Hee Kim contributed equally to this work.

c) 2020 The Korean Academy of Medical Sciences.

This is an Open Access article distributed under the terms of the Creative Commons Attribution Non-Commercial License (https:// creativecommons.org/licenses/by-nc/4.0/) which permits unrestricted non-commercial use, distribution, and reproduction in any medium, provided the original work is properly cited.

ORCID iDs

Chung Mo Koo (ID)

https://orcid.org/0000-0003-1434-6988

Se Hee Kim (iD

https://orcid.org/0000-0001-7773-1942 Joon Soo Lee (1)

https://orcid.org/0000-0001-9036-9343

\title{
Cannabidiol for Treating Lennox- Gastaut Syndrome and Dravet Syndrome in Korea
}

\author{
Chung Mo Koo (D),,$^{*}$ Se Hee Kim (D),,$^{2^{*}}$ Joon Soo Lee $\left(\mathbb{D},{ }^{2}\right.$ Byung-Joo Park (D), \\ Hae Kook Lee $\mathbb{1}^{10,5}$ Heung Dong Kim (1), ${ }^{2}$ and Hoon-Chul Kang (1) ${ }^{2,4}$
}

'Division of Pediatric Emergency, Department of Pediatrics, Severance Children's Hospital, Yonsei University College of Medicine, Seoul, Korea

${ }^{2}$ Division of Pediatric Neurology, Department of Pediatrics, Epilepsy Research Institute, Severance Children's Hospital, Yonsei University College of Medicine, Seoul, Korea

${ }^{3}$ Department of Preventive Medicine, Seoul National University College of Medicine, Seoul, Korea

${ }^{4}$ National Academy of Medicine of Korea, Seoul, Korea

${ }^{5}$ Department of Psychiatry, College of Medicine, The Catholic University of Korea, Seoul, Korea

\section{ABSTRACT}

Background: For the first time in Korea, we aimed to study the efficacy and safety of cannabidiol (CBD), which is emerging as a new alternative in treating epileptic encephalopathies.

Methods: This study was conducted retrospectively with patients between the ages of 2-18 years diagnosed with Lennox-Gastaut syndrome (LGS) or Dravet syndrome (DS) were enrolled from March to October 2019, who visited outpatient unit at 3 and 6 months to evaluate medication efficacy and safety based on caregiver reporting. Additional evaluations, such as electroencephalogram and blood tests, were conducted at each period also. CBD was administered orally at a starting dose of $5 \mathrm{mg} / \mathrm{kg} / \mathrm{day}$, and was maintained at $10 \mathrm{mg} / \mathrm{kg} / \mathrm{day}$. Results: We analyzed 34 patients in the LGS group and 10 patients in the DS group between the ages of 1.2-15.8 years. In the 3-month evaluation, the overall reduction of seizure frequency in the LGS group was $52.9 \%$ ( $>50 \%$ reduction in $32.3 \%$ of the cases), and $29.4 \%$ in the 6-month evaluation (more than $50 \%$ reduction in $20.6 \%$ ). In DS group, the reduction of seizure frequency by more than $50 \%$ was $30 \%$ and $20 \%$ in the 3 -month and 6 -month evaluation, respectively. Good outcomes were defined as the reduction of seizure frequency by more than $50 \%$ and similar results were observed in both LGS and DS groups. Adverse events were reported in $36.3 \%$ of total patients of which most common adverse events were gastrointestinal problems. However, no life-threatening adverse event was reported in both LGS and DS during the observation period.

Conclusion: In this first Korean study, CBD was safe and tolerable for use and could be expected to potentially reduce the seizure frequency in pediatric patients with LGS or DS.

Keywords: Cannabidiol; Lennox Gastaut Syndrome; Dravet Syndrome

\section{INTRODUCTION}

Epilepsy is associated with cognitive dysfunction and behavioral disorders. Uncontrolled seizures often affect one's quality of life, especially when they occur at a young age..$^{1-5}$ In particular, in epileptic encephalopathies such as Lennox-Gastaut syndrome (LGS) and Dravet syndrome (DS), 
Byung-Joo Park (iD)

https://orcid.org/0000-0003-4630-4942

Hae Kook Lee iD

https://orcid.org/0000-0002-3941-2980

Heung Dong Kim (D)

https://orcid.org/0000-0002-8031-7336

Hoon-Chul Kang (iD

https://orcid.org/0000-0002-3659-8847

Funding

This study was supported by the National Academy of Medicine of Korea. This study was supported by the Life Insurance Social Contribution Committee (2020). This study was supported by Kyobo Life Insurance Co., Ltd. (2020).

Disclosure

The authors have no potential conflicts of interest to disclose.

\section{Author Contributions}

Conceptualization: Koo CM, Kim SH, Lee JS, Kim HD, Kang HC. Methodology: Koo CM, Kim SH, Lee JS, Kim HD, Kang HC. Writing - original draft: Koo CM, Kim SH. Writing - review \& editing: Koo CM, Kim SH, Lee JS, Park BJ, Lee HK, Kim HD, Kang HC epileptic activity causes severe cognitive and behavioral disorders to worsen over time. ${ }^{6}$

Purified cannabidiol (CBD) (Epidiolex ${ }^{\circledR}$; GW Pharmaceuticals, Cambridge, UK) is a new drug that can be used to treat drug-resistant epilepsy, ${ }^{7}$ as its administration can reduce seizure frequency. Mild adverse events such as somnolence and gastrointestinal (GI) symptoms have been reported. ${ }^{7-10}$ After randomized trials assessing CBD use in LGS and DS were conducted, the United States Food and Drug Administration approved the prescription of CBD for both epileptic encephalopathies in 2018.11,12

CBD exerts anti-seizure effects, while another component of cannabis, $\Delta 9$-tetrahydrocannabinol (THC), has psychoactive functions.13,14 In Korea, due to the addictive nature of THC, even CBD was considered an illegal drug until March 2019. The efforts of the media, patient groups, and non-governmental organizations informed the public that CBD medication contained a very small amount of THC, with a very low potential for addiction and psychoactive effects. These acts led to the approval of CBD by the Ministry of Food and Drug Safety for the treatment of DS or LGS in those over two years of age.

This study was performed to evaluate the tolerability and efficacy of CBD in children with LGS or DS for the first time in Korea.

\section{METHODS}

\section{Patients and study design}

Patients who initiated CBD treatment from March 2019 to October 2019 and who were between the ages of 2 and 18 years and diagnosed with of DS or LGS were included in this study. All patients diagnosed with DS met the clinical diagnostic criteria, including an early onset of febrile or vaccination related seizures, developmental regression in relation to the seizures, and drug-resistant epilepsy. All had pathogenic SCN1A (sodium channel, voltage-gated, type I, alpha subunit) mutations. All patients diagnosed with LGS exhibited impaired intellectual function and multiple mixed-type seizures, as well as characteristic electroencephalography (EEG) patterns showing generalized sharp and slow waves (GSSW) or generalized paroxysmal fast activities (PFA). This study was conducted retrospectively and evaluated the medication efficacy and safety through outpatient visits at 3 and 6 months based on caregiver reporting. Patients received Epidiolex ${ }^{\circledR}$, manufactured by GW Pharmaceuticals, which contained CBD $(100 \mathrm{mg} / \mathrm{mL})$. CBD was administered orally at a starting dose of $5 \mathrm{mg} / \mathrm{kg} /$ day, in addition to baseline antiepileptic drug therapy. After 1 week, the dosage was up-titrated by $5 \mathrm{mg} / \mathrm{kg} /$ day and was maintained at $10 \mathrm{mg} / \mathrm{kg} /$ day (Fig. 1).

\section{Assessment of efficacy}

The efficacy of CBD was evaluated by comparing the mean frequency of motor seizures experienced per month, defined by the tonic, clonic, and atonic components, before and after the administration of medication. The change in the seizure frequency was expressed as a percentage that was calculated as [(seizure frequency per month) - (seizure frequency at baseline)]/(seizure frequency at baseline) $\times 100$. Each patient's response to CBD was categorized as one of the following 4 responses: 1) the patient became seizure-free, 2) the frequency of seizures was reduced by more than $50 \%, 3$ ) the frequency of seizures was reduced by less than $50 \%$, or 4 ) there was no effect. A good outcome was defined as the seizure frequency being reduced by more than $50 \%$, including being seizure-free. 


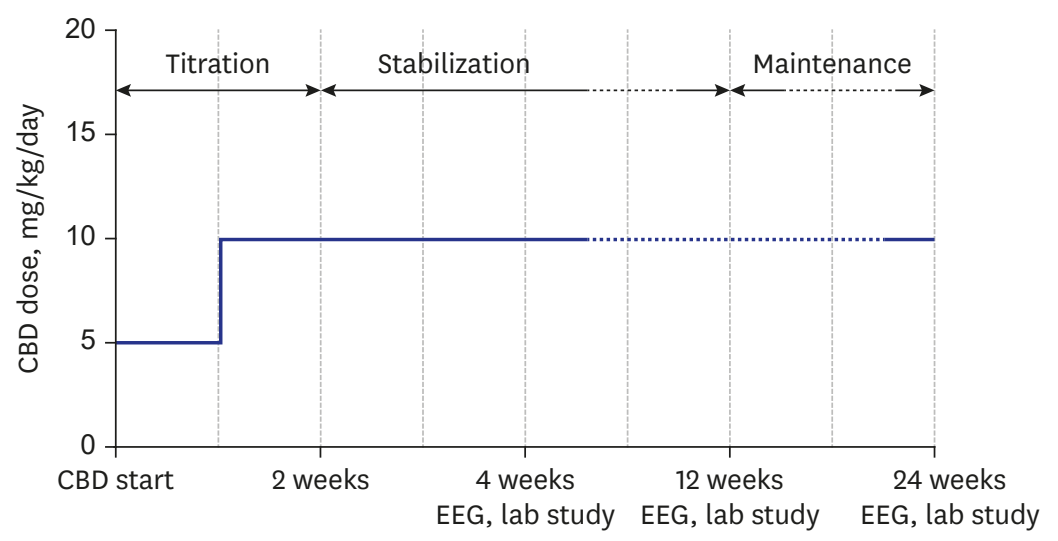

Fig. 1. Cannabidiol schedule.

$\mathrm{CBD}=$ cannabidiol, $\mathrm{EEG}=$ electroencephalogram.

The additional effect of CBD was evaluated based on comparisons of the EEGs performed during each period, and each assessment was performed for 30 minutes or more. Each scalp electrode was placed in accordance with an international 10-20 system. The diagnosis was based on the characteristic interictal and ictal patterns of the EEG.15 In the LGS group, we evaluated whether the EEG improved at the 3-and 6-month visits, and the criteria were based on the persistence of the EEG characteristics of LGS or the prevalence of the epileptiform pattern. ${ }^{16}$

\section{Assessment of safety}

All safety results in this report were evaluated over the entire follow-up period. Patients or caregivers documented the adverse events at each visit. Safety was assessed through laboratory studies, including complete blood counts, liver function tests, and vital signs at baseline and at the time of the outpatient clinic visits. The appearance of adverse events was resolved by dose reduction or discontinuation of treatment.

\section{Analyses}

An intention-to-treat analysis was conducted to assess CBD efficacy. Data processing and analysis were performed with Statistical Package for the Social Sciences version 25 (IBM Co., Armonk, NY, USA). $P$ values $<0.05$ were regarded as statistically significant. All numbers were rounded to 2 decimal places. The statistical analyses included the independent paired-sample $t$-test and Fisher's exact test, and Pearson's $\chi^{2}$ test to compare the variables and prognosis.

\section{Ethics statement}

This study was approved by the Institutional Review Board of Severance Hospital (approval No.: 4-2020-0120). Informed consent was waived due to the retrospective nature of the study.

\section{RESULTS}

\section{Study population}

During the study period, 44 patients were managed with CBD, 34 of whom were in the LGS group and 10 of whom were in the DS group. One patient from the LGS group was excluded due to follow-up loss. One patient from the DS group was excluded due to the occurrence of an adverse event before the 3-month evaluation. After the 3-month evaluation, 6 patients in the LGS group were excluded from the study due to dissatisfaction with the treatment effect, 


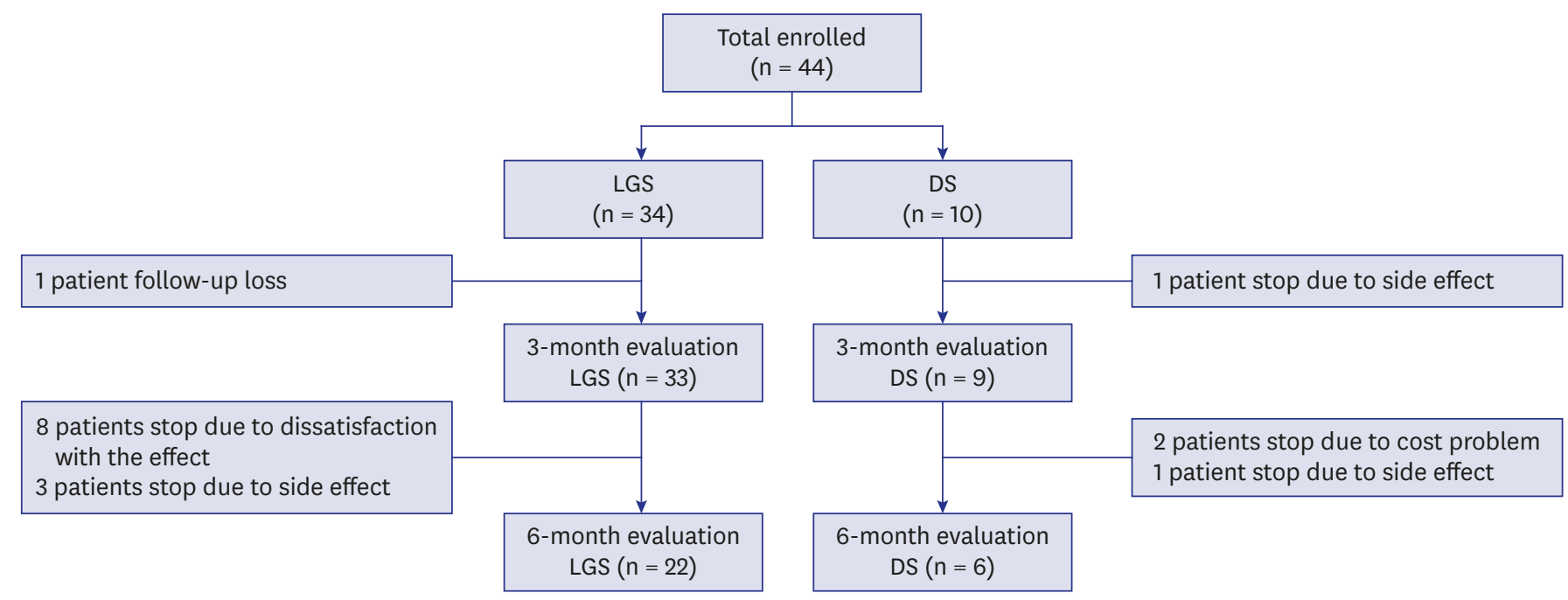

Fig. 2. Study participants' flow chart.

LGS = Lennox-Gastaut syndrome, DS = Dravet syndrome.

and 3 patients were excluded because of the development of adverse events. In the DS group, 1 patient was excluded due to adverse events and 2 were excluded due to financial constraints (Fig. 2).

The ages of the patients included in this study ranged from 1.2 to 15.8 years (mean age = 5.1 years), with 28 males (63.6\%) and 16 females (36.4\%). There were 5 patients $(11.4 \%)$ undergoing diet therapy. The most frequently used antiepileptic drug at the time of the study was valproic acid (VPA) at an average dose of $22.2 \mathrm{mg} / \mathrm{kg} /$ day, followed by levetiracetam at an average dose of $20.3 \mathrm{mg} / \mathrm{kg} /$ day. Stiripentol was used only in DS patients. Patient characteristics are shown in Table 1.

Table 1. Baseline characteristics

\begin{tabular}{lc}
\hline Characteristics & Patients receiving CBD $(\mathrm{n}=44)$ \\
\hline Male:female & $28(63.6): 16(36.4)$ \\
Mean age, $y r$ & $5.1(\max =15.8$, min $=1.2$, IQR $=2.9-6.5)$ \\
Epilepsy syndrome & $34(77.3)$ \\
$\quad$ Lennox-Gastaut syndrome & $10(23.3)$ \\
$\quad$ Dravet syndrome & $3(1-5)$ \\
Concomitant use of antiepileptic drugs & $36(81.8)$ \\
Antiepileptic drugs used & $17(38.6)$ \\
$\quad$ Valproic acid & $12(27.3)$ \\
$\quad$ Clobazam & $5(11.4)$ \\
$\quad$ Topiramate & \\
Stiripentol & $5(11.4)$ \\
Other treatment in progress at the time of CBD use & $2(4.5)$ \\
Diet therapy & \\
Steroids & \\
EEG patterns before cannabidiol treatment & $16(36.3)$ \\
Paroxysmal fast activities & $17(38.6)$ \\
Generalized sharp and slow waves & $22(50)$ \\
Abnormal brain MRI findings &
\end{tabular}

Data are presented as median (range) or number (\%) unless otherwise indicated.

$\mathrm{CBD}$ = cannabidiol, IQR = interquartile range, $\mathrm{EEG}$ = electroencephalogram, $\mathrm{MRI}$ = magnetic resonance imaging. ${ }^{a}$ All diet therapies used in this study were the modified Atkins diet; ${ }^{\text {b} A l l ~ u s e d ~ d e f l a z a c o r t . ~}$ 


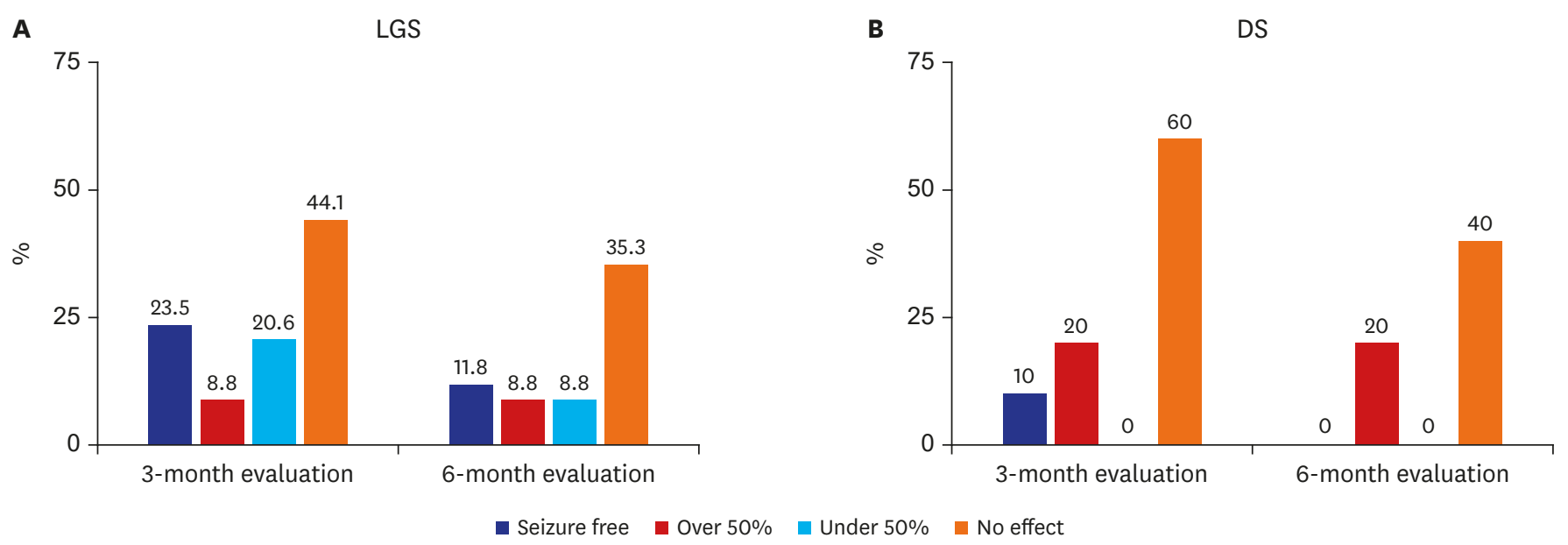

Fig. 3. Outcome of seizures with cannabidiol treatment. (A) At 3 and 6 months of evaluation in LGS. (B) At 3 and 6 months of evaluation in DS. LGS = Lennox-Gastaut syndrome, DS = Dravet syndrome.

\section{Efficacy in LGS}

When outcomes were observed in the LGS group, a reduction in seizure frequency was observed in $52.9 \%$ of the patients at 3 months and in $29.4 \%$ at 6 months. Eight patients $(23.5 \%)$ were seizure-free at the 3 -month evaluation, 3 patients $(8.8 \%)$ had a reduction in seizure frequency of more than $50 \%$, and 7 patients $(20.6 \%)$ showed a reduction in seizure frequency of less than $50 \%$. It can be stated that the result was good in $32.3 \%$ of patients. At the 6-month evaluation, 4 patients $(11.8 \%)$ were seizure-free and 3 patients $(8.8 \%)$ showed more than a $50 \%$ reduction in seizure frequency; thus, $20.6 \%$ of the patients had good outcomes (Fig. 3A).

When evaluating the initial EEGs of LGS patients, 16 cases showed GSSW or PFA, characteristic of LGS. The outcomes at the 3-and 6-month evaluations were investigated. At the 3-month evaluation, good outcomes were observed as there were more cases with an initial EEG that did not show the PFA, but at the 6-month evaluation, the opposite was observed. In the case of the GSSW pattern, good outcomes were obtained, with no cases showing the pattern at either the 3- or the 6-month evaluations; however, there was no statistically significant change in EEG activity after the administration of CBD.

When compared with the baseline EEG, most of the patients who showed no improvement at the 3 -month evaluation $(P$ value $=0.017)$ and the 6 -month evaluation $(P$ value $=0.009)$ were determined to have had a "no effect" outcome.

\section{Efficacy in DS}

In the DS group, the "no effect" outcome was observed at a higher frequency at both the 3and 6-month evaluations than it was in the LGS group. At the 3-month evaluation, 1 patient $(10.0 \%)$ was seizure-free, 2 patients $(20.0 \%)$ showed a more than $50 \%$ reduction in seizure frequency, and $60.0 \%$ of the enrolled patients experienced no effect. None of the patients were "seizure-free" at the 6-month evaluation, and only $2(20.0 \%)$ showed a reduction in seizure frequency of $50 \%$ or more. At the 6-month evaluation, a "no effect" outcome was observed in $66.7 \%$ of patients (Fig. 3B). 
Table 2. Adverse events of cannabidiol

\begin{tabular}{lc}
\hline Adverse events & Values \\
\hline Gastrointestinal problems & $7(15.9)$ \\
Vomiting or diarrhea & $4(9.1)$ \\
Decreased appetite & $1(2.3)$ \\
Liver enzyme elevation & $1(2.3)$ \\
Acute pancreatitis & $1(2.3)$ \\
Drowsiness & $3(6.8)$ \\
Reduction of AED dosages & $2(4.5)$ \\
Reduction of CBD dosage & $1(2.3)$ \\
Behavioral changes & $5(11.4)$ \\
Increased seizure frequency & $2(4.5)$ \\
Skin rash & $1(2.3)$ \\
\hline
\end{tabular}

Values are presented as number (\%).

AED = antiepileptic drug, CBD = cannabidiol.

\section{Safety}

No life-threatening adverse event was reported in the LGS and DS groups during the observation period. However, adverse events were reported in 16 (36.3\%) of the enrolled patients, and GI problems were the most frequent adverse event in 7 cases (15.9\%); among them, mild liver enzyme elevation was observed in 1 case, whereas most included vomiting or diarrhea. Five cases (11.4\%) experienced behavioral changes, such as irritability, hyperactivity, excessive alertness, and sleep disturbances (Table 2). In the DS group, 2 cases had increased seizure frequency, which warranted the discontinuation of the medication. The other adverse events necessitating the cessation of the drug were as follows: 1 case of drowsiness, 1 case of GI problems with acute pancreatitis, and 1 case of behavioral changes.

\section{DISCUSSION}

This study evaluated the efficacy and safety of CBD administration for treating LGS and DS for the first time in Korea. A good treatment outcome was observed in $31.8 \%$ of patients at the 3-month evaluation and in 20.5\% at the 6-month evaluation, including both the LGS and DS groups. This positive response was lower than the $36 \%-57 \%$ positive response within 6 months shown previously by studies that did not distinguish between the patient groups. ${ }^{7,8,17}$

Among the LGS patients, a good outcome was observed in 32.4\% of cases at the 3-month evaluation and in $20.6 \%$ at the 6 -month evaluation, which was similar to the percentage of all patients who showed good treatment outcomes overall. When comparing the effects of CBD treatment over a similar period in other studies that assessed LGS patients, good outcomes were noted in $33 \%-44 \%$ of cases. 11,18 In our study, the percentage of good outcomes was slightly lower; however, it was within a similar range. According to a study by Thiele et al.11 conducted in 2018, when comparing the LGS group using CBD and a patient group receiving a placebo, the monthly frequency of drop attacks decreased by $43.9 \%$ in those receiving $\mathrm{CBD}$, compared to a reduction of only $21.8 \%$ in the placebo group. The total monthly seizure frequency decreased by $41.2 \%$ in the CBD group compared to a $13.7 \%$ decrease in the placebo group. Moreover, Thiele et al. ${ }^{11}$ documented that caregivers reported an improved condition in the CBD group that was $24 \%$ more than that of the placebo group. From these results, it appears that the use of CBD in LGS patients can reduce the frequency of seizures, including drop attacks, which can be a major obstacle in everyday life; thus, CBD treatment provides the opportunity for a good treatment outcome by reducing the seizure frequency by more than $50 \%$ in LGS patients. 
In the case of the DS group, as the number of patients enrolled was small, an accurate evaluation was difficult. Upon reviewing previous studies, the reduction in seizure frequency by more than $50 \%$ was found to be about $43 \%, 12,19$ whereas, in our research, it was $30.0 \%$ at the 3-month evaluation. We evaluated the EEG changes and outcomes as we did with the LGS, but the changes do not seem to be relevant. Devinsky et al.12 reported that the monthly frequency of motor seizures experienced by the DS group using CBD decreased by a median of $6.5 \%$, compared to a reduction of $0.8 \%$ in the placebo group. However, patients with LGS improved not only in terms of motor seizures but also in terms of seizures of other types, whereas DS patients showed no significant improvement in seizures overall, except for motor seizures. 12

According to a recently published study that conducted a comparison of outcomes and CBD dosages in a DS patient group, the number of cases experiencing a reduction in seizure frequency of at least $50 \%$ was higher in the CBD 20 group ( $20 \mathrm{mg} / \mathrm{kg} / \mathrm{day}$ ) than in the CBD 10 group $(10 \mathrm{mg} / \mathrm{kg} / \mathrm{day})$. On the contrary, more individuals experienced a reduction in seizure frequency of more than $75 \%$ in the CBD 10 group than in the CBD 20 group. ${ }^{19}$ In a study related to LGS treatment with CBD published in 2018, a reduction in the frequency of seizures by more than $50 \%$ during the research period was observed for $36 \%$ of patients in the CBD 10 group and 39\% of patients in the CBD 20 group. Moreover, the median percent reduction in monthly seizure frequency tended to decrease further at a higher dosage of CBD, except for non-drop attacks. ${ }^{18}$

Usually, adverse events related to CBD administration are mild, and the most common adverse events are drowsiness and GI problems such as diarrhea. In rare cases, serious adverse events occur that may require hospital admission for treatment, such as status epilepticus or extrapyramidal symptoms. ${ }^{7,8,20}$ However, in the case of status epilepticus, the causal relationship with CBD is unclear because most enrolled patients were those with refractory epilepsy, such as LGS or DS. In our study, similar to others, the adverse events reported included high rates of GI problems, such as diarrhea and inadequate appetite, which can be expected, as the current formulation of CBD in use is in the form of an oil.

Drowsiness and other adverse events, such as elevated liver enzymes, that have been reported in some studies ${ }^{7}$ were thought to be essential for confirming the interaction between CBD and antiepileptic drugs such as VPA and clobazam (CLB) through further research; the reason for this is that the metabolism of CBD by the liver can inhibit cytochrome P450. This affects the metabolism of some antiepileptic drugs; in the case of CLB, it can increase the serum level of N-desmethylclobazam, which is an active metabolite of CLB.8,21-24

There were some limitations in this study. For example, this study was performed with a small number of enrolled patients from a single institution; primary data collection was performed through the assessment of caregivers' reports; and adverse events were investigated retrospectively and may not have been accurate. In addition, there may have been a selection bias.

However, this study is still meaningful, as it is the first pilot study to be conducted in Korea, showing that CBD can be used effectively and safely at a dose of $10 \mathrm{mg} / \mathrm{kg} /$ day for treating children of Asian ethnicity. In the future, we plan to conduct a well-designed, randomized, controlled clinical trial to evaluate CBD efficacy, safety, and drug interactions. 


\section{REFERENCES}

1. Donner EJ. Opportunity gained, opportunity lost: treating pharmacoresistant epilepsy in children. Epilepsia 2013;54 Suppl 2:16-8. PUBMED | CROSSREF

2. Cilio MR, Thiele EA, Devinsky O. The case for assessing cannabidiol in epilepsy. Epilepsia 2014;55(6):787-90. PUBMED | CROSSREF

3. Berg AT, Zelko FA, Levy SR, Testa FM. Age at onset of epilepsy, pharmacoresistance, and cognitive outcomes: a prospective cohort study. Neurology 2012;79(13):1384-91. PUBMED | CROSSREF

4. O'Connell BK, Gloss D, Devinsky O. Cannabinoids in treatment-resistant epilepsy: a review. Epilepsy Behav 2017;70(Pt B):341-8. PUBMED | CROSSREF

5. Koo CM, Kang HC. Could cannabidiol be a treatment option for intractable childhood and adolescent epilepsy? J Epilepsy Res 2017;7(1):16-20. PUBMED | CROSSREF

6. Berg AT, Berkovic SF, Brodie MJ, Buchhalter J, Cross JH, van Emde Boas W, et al. Revised terminology and concepts for organization of seizures and epilepsies: report of the ILAE Commission on Classification and Terminology, 2005-2009. Epilepsia 2010;51(4):676-85. PUBMED | CROSSREF

7. Devinsky O, Marsh E, Friedman D, Thiele E, Laux L, Sullivan J, et al. Cannabidiol in patients with treatment-resistant epilepsy: an open-label interventional trial. Lancet Neurol 2016;15(3):270-8. PUBMED | CROSSREF

8. Klotz KA, Grob D, Hirsch M, Metternich B, Schulze-Bonhage A, Jacobs J. Efficacy and tolerance of synthetic cannabidiol for treatment of drug resistant epilepsy. Front Neurol 2019;10:1313. PUBMED | CROSSREF

9. Hussain SA, Zhou R, Jacobson C, Weng J, Cheng E, Lay J, et al. Perceived efficacy of cannabidiol-enriched cannabis extracts for treatment of pediatric epilepsy: a potential role for infantile spasms and LennoxGastaut syndrome. Epilepsy Behav 2015;47:138-41. PUBMED | CROSSREF

10. Mitelpunkt A, Kramer U, Hausman Kedem M, Zilbershot Fink E, Orbach R, Chernuha V, et al. The safety, tolerability, and effectiveness of PTL-101, an oral cannabidiol formulation, in pediatric intractable epilepsy: a phase II, open-label, single-center study. Epilepsy Behav 2019;98(Pt A):233-7. PUBMED | CROSSREF

11. Thiele EA, Marsh ED, French JA, Mazurkiewicz-Beldzinska M, Benbadis SR, Joshi C, et al. Cannabidiol in patients with seizures associated with Lennox-Gastaut syndrome (GWPCARE4): a randomised, doubleblind, placebo-controlled phase 3 trial. Lancet 2018;391(10125):1085-96. PUBMED | CROSSREF

12. Devinsky O, Cross JH, Wright S. Trial of cannabidiol for drug-resistant seizures in the Dravet syndrome. $N$ Engl J Med 2017;377(7):699-700. PUBMED | CROSSREF

13. Lutz B. On-demand activation of the endocannabinoid system in the control of neuronal excitability and epileptiform seizures. Biochem Pharmacol 2004;68(9):1691-8. PUBMED | CROSSREF

14. Pertwee RG, Cascio MG. Known pharmacological actions of delta-9-tetrahydrocannabinol and of four other chemical constituents of cannabis that activate cannabinoid receptors. In: Pertwee RG, editor. Handbook of Cannabis. Oxford: Oxford University Press; 2014.

15. Bureau M, Genton P, Dravet C, Delgado-Escueta AV, Tassinari CA, Thomas P, et al. Epileptic Syndromes in Infancy, Childhood and Adolescence. 5th ed. Montrouge: John Libbey Eurotext; 2012.

16. Kane N, Acharya J, Benickzy S, Caboclo L, Finnigan S, Kaplan PW, et al. A revised glossary of terms most commonly used by clinical electroencephalographers and updated proposal for the report format of the EEG findings. Revision 2017. Clin Neurophysiol Pract 2017;2:170-85. PUBMED | CROSSREF

17. Goldenholz DM, Moss R, Scott J, Auh S, Theodore WH. Confusing placebo effect with natural history in epilepsy: a big data approach. Ann Neurol 2015;78(3):329-36.

PUBMED | CROSSREF

18. Devinsky O, Patel AD, Cross JH, Villanueva V, Wirrell EC, Privitera M, et al. Effect of cannabidiol on drop seizures in the Lennox-Gastaut syndrome. N Engl J Med 2018;378(20):1888-97.

PUBMED | CROSSREF 
19. Miller I, Scheffer IE, Gunning B, Sanchez-Carpintero R, Gil-Nagel A, Perry MS, et al. Dose-ranging effect of adjunctive oral cannabidiol vs placebo on convulsive seizure frequency in Dravet syndrome: a randomized clinical trial. JAMA Neurol 2020;77(5):613-21. PUBMED | CROSSREF

20. Szaflarski JP, Bebin EM, Comi AM, Patel AD, Joshi C, Checketts D, et al. Long-term safety and treatment effects of cannabidiol in children and adults with treatment-resistant epilepsies: expanded access program results. Epilepsia 2018;59(8):1540-8. PUBMED | CROSSREF

21. Stout SM, Cimino NM. Exogenous cannabinoids as substrates, inhibitors, and inducers of human drug metabolizing enzymes: a systematic review. Drug Metab Rev 2014;46(1):86-95. PUBMED | CROSSREF

22. Patsalos PN, Perucca E. Clinically important drug interactions in epilepsy: general features and interactions between antiepileptic drugs. Lancet Neurol 2003;2(6):347-56. PUBMED | CROSSREF

23. Geffrey AL, Pollack SF, Bruno PL, Thiele EA. Drug-drug interaction between clobazam and cannabidiol in children with refractory epilepsy. Epilepsia 2015;56(8):1246-51. PUBMED | CROSSREF

24. Morrison G, Crockett J, Blakey G, Sommerville K. A phase 1, open-label, pharmacokinetic trial to investigate possible drug-drug interactions between clobazam, stiripentol, or valproate and cannabidiol in healthy subjects. Clin Pharmacol Drug Dev 2019;8(8):1009-31.

PUBMED | CROSSREF 